Postprint of Food Microbiology Volume 69, February 2018, Pages 33-42

DOI: https://doi.org/10.1016/j.fm.2017.07.010

\title{
Genotyping, identification and multifunctional features of yeasts associated to Bosana naturally black table olive fermentations
}

Cinzia Porru ${ }^{1}$, Francisco Rodríguez-Gómez ${ }^{2}$, Antonio Benítez-Cabello², Rufino Jiménez-Díaz ${ }^{2}$, Giacomo Zara ${ }^{1}$, Marilena Budroni ${ }^{1, *}$, Ilaria Mannazzu ${ }^{1}$, Francisco Noé Arroyo-López ${ }^{2}$

${ }^{1}$ Dipartimento di Agraria. University of Sassari. Viale Italia 39. Sassari, I-07100, Italy.

${ }^{2}$ Food Biotechnology Department. Instituto de la Grasa (CSIC). University Campus Pablo de Olavide. Building 46. Crta. Utrera Km 1. 41013. Seville, Spain.

Running Title: Yeasts isolated from Bosana olive fermentations

*Corresponding author: Marilena Budroni, PhD. Dipartimento AGRARIA, University of Sassari. E-mail: mbudroni@uniss.it 


\section{Abstract}

Directly brined black table olives of Bosana variety are a traditional food product of Sardinia island (Italy), spontaneously fermented by yeasts among other microorganisms. However, as far as we know, the identification, biotechnological and probiotic potential of this yeast community has not been investigated yet. In this work, a total of 72 yeast isolates previously obtained from Bosana olive brines were first genotyped by Random Amplified Polymorphic DNA (RAPD-PCR) analysis with primer M13, and then identified by sequencing of D1/D2 domains of rDNA 26S gene. The dominant species were Wickerhamomyces anomalus and Nakazawaea molendini-olei, albeit Candida diddensiae, Candida boidinii, Zygotorulaspora mrakii, and Saccharomyces cerevisiae were also present in lower proportions. For the different biotypes of yeasts obtained, the multivariate analysis of their technological (esterase, lipase and $\beta$-glucosidase activities, growth in presence of oleuropein, resistance and susceptibility to $\mathrm{NaCl}$ ) and probiotic (removal of cholesterol, gastric and pancreatic digestions, biofilms assays alone and in co-culture with Lactobacillus pentosus) features, showed that W. anomalus Wa1 exhibited the best technological characteristics, while $S$. cerevisiae $\mathrm{Sc} 24$ and $C$. boidinii Cb60 showed promising probiotic features. Therefore, they may have potential application as multifunctional starters, alone or in combination with lactic acid bacteria, during olive processing, albeit further studies are necessary to validate these results.

Keywords: Biofilms; olive fermentations; Multifunctional starter; Yeast community 


\section{Introduction}

Table olives are one of the most important and well-known fermented vegetables of the food industry, with an estimated world production above 2.5 million tonnes per year (IOC, 2017). The main producing area is around the Mediterranean Basin, with an outstanding contribution of the European Union (EU) (around 30\% of production). Within it, Spain is the leading country, followed by Greece and Italy (IOC, 2017). The Mediterranean diet pyramid recommends a daily intake of table olives due to the nutritional benefits associated with this fermented vegetable (Bach-Faig et al., 2011; Arroyo-López et al., 2016). Particularly, consumers are increasingly demanding more traditional and natural homemade olive preparations.

Olive fruit contains a bitter component (oleuropein) that makes it unpalatable. Thus, the main goal of table olive processing is to remove the natural bitterness of fresh fruit. In the case of natural olives, not subjected to "lye" treatment as occur in Bosana olive specialty, debittering is obtained just by immersion of the harvested drupes in brine. In this case, the removal of the fruit bitterness is produced by hydrolysis of oleuropein, due, among other factors, to esterase or $\beta$-glucosidase activities by microorganisms (Tassou et al., 2002; Arroyo-López et al., 2012), or by diffusion of this compound from fruits to the surrounding brine (Garrido-Fernandez et al., 1997). Therefore, the process is rather slow.

Lactic acid bacteria (LAB) play an important role in table olive fermentations (Garrido-Fernandez et al., 1997). In fact, they produce antimicrobial substances (bacteriocins), and lactic acid originated from the fermentation of sugars, resulting in pH decrease that enhances table olives safety (Ruiz-Barba and Jimenez-Diaz, 1995; 
Corsetti et al., 2012). Especially, Lactobacillus pentosus is a species with a significant relevance in table olive production because of its predominance during fermentative process and use as starter culture (Tofalo et al., 2014; Martorana et al., 2017; Rodríguez-Gómez et al., 2017). However, sometimes, yeasts can be the dominant microorganism during table olive fermentation, especially when LAB are inhibited due to the presence of phenolic compounds or the high salt and low $\mathrm{pH}$ levels obtained during the fermentation process (Ruiz-Barba et al., 1993; Garrido-Fernández et al., 1997). Thereby, they have also a considerable influence during olive processing, acting as desirable or undesirable microorganisms (Arroyo-López et al., 2012). For many years, the search for starters with application in olive fermentation was focused exclusively on the activity of LAB. However, in the last years, different works and reviews have considered the beneficial effects that the use of yeasts may have during olive processing due to their biotechnological and probiotic potential (Silva et al., 2011; Arroyo-López et al., 2012; Bevilacqua et al., 2012, 2013, 2015; Tofalo et al., 2013; Bleve et al., 2015; Bonatsou et al., 2015; De Angelis et al., 2015; Tufariello et al., 2015). For this reason, yeasts may be exploited in table olive processing as multifunctional starters, particularly in the case of natural olives which spontaneous processing is usually performed by a microbiota mainly composed of yeasts (GarridoFernández et al., 1997).

The present paper has the double objective of i) expanding the knowledge of the yeast community associated to traditional table olives preparations from Sardinia island, and ii) determining their biotechnological and probiotic potential for their use as multifunctional starters.

\section{Material and methods}




\subsection{Strains isolation}

The study was conducted with a collection of 72 yeasts previously isolated from brines of traditional spontaneous fermentations of Bosana fruits (Pinna, 2005), a olive variety largely cultivated in Northern Sardinia (Italy). Fruits were harvested during the 2003/2004 season at black ripening stage in the same olive ground. After sorting, sizing, and washing, the olives were dried and placed into barrels (20 L capacity), which were filled with $10.5 \mathrm{~kg}$ of fruits and $9.5 \mathrm{~L}$ of $60 \mathrm{~g} / \mathrm{L} \mathrm{NaCl}$ brine (ratio brine/drupe of approximately 1:1). Yeasts were isolated as described by Hernandez et al. (2007) during the fermentation process at $0,1,2,5,10,15,22,30,60,90,120,150$, and 180 days. Then, they were stored $\left(-80^{\circ} \mathrm{C}\right)$ in Dipartimento di Agraria of Sassary University (Italy) in YM broth medium (Difco ${ }^{\mathrm{TM}}$, Becton and Dickinson Company, Sparks, MD, USA) supplemented with $20 \%$ glycerol for further analysis.

\subsection{Genotyping and identification of yeast population}

Genomic DNA extraction was performed by the procedure described by SensesErgul et al. (2012). The extracted and purified DNA was quantified using the internal protocol incorporated in the Spectrostar Nano spectrophotometer (BMG LABTECH, Germany) through absorbance reading at $260 \mathrm{~nm}$.

Yeast genotyping was performed by Random Amplified Polymorphic DNA (RAPD)-PCR analysis with M13 primer according to the procedures described by Andrighetto et al. (2000). RAPD products were visualized with a gel analyzer model EnduroTM GDS (Labnet International, Inc., USA). The fingerprints obtained for the different yeast isolates were captured and analysed using Bionumerics 6.6 software (Applied Maths, Belgium). Dendrogram for clustering comparison was built with 
UPGMA (Unweighted Pair Group Method) method and Pearson correlation. Reproducibility and sensibility of the technique were evaluated by comparing the profiles of the strain Wickerhamomyces anomalus TOMC-Y45 obtained from seven different DNA extractions, PCR and agarose gels.

To corroborate the clustering analysis and for identification of the yeasts at the species level, sequencing of D1/D2 domains of 26S rDNA was performed for all the yeast isolates using NL1 and NL4 primers (Kurtzman and Robnett, 1998) and the protocols described by Arroyo-López et al. (2006). Purification of the PCR products for sequencing was carried out using the Isolate DNA kit (Bioline, Taunton, USA) according to the manufacturer's instructions, and sequenced by the StabVida Sequencing Services (Lisbon, Portugal). The alignment of sequences obtained was performed with software MEGA 5.0 (Molecular Evolution Genetic Analysis) and then compared with sequences deposited in the NCBI (National Center for Biotechnology Information, USA) GenBank database using the BLAST (Basic Local Alignment Search Tool) software.

\subsection{Evaluation of technological features}

The following technological assays were performed (in triplicate) using one representative strain chosen randomly from each different cluster of the dendrogram: i) the modelling of susceptibility (NIC) and resistance (MIC) to $\mathrm{NaCl}$ in $\mathrm{YM}$ broth medium $(\mathrm{pH} 4.5)$, supplemented with different $\mathrm{NaCl}$ concentrations $(0,10,30,50,70$, $80,90,100,110,120,130,150$, and $180 \mathrm{~g} / \mathrm{L})$, was determined by the application of a dose/response model using a modified Gompertz equation according to procedures described by Bonatsou et al. (2015); ii) extracellular and cellular $\beta$-glucosidase, esterase and lipase enzymatic activities were measured spectrophotometrically (absorbance at 
$410 \mathrm{~nm}$ ) based on the ability of these enzymes to release p-nitrophenol from different chromogenic substrate and expressed as $\mathrm{nmol} \mathrm{ml} / \mathrm{h}$ according to procedures described by Rodríguez Gómez et al. (2012); and iii) growth in the presence of oleuropein was assayed in YNB medium (Difco) at $\mathrm{pH} 4.5$ supplemented with $1.0 \%$ (wt/vol) of oleuropein (Extrasynthese, Lyon, France) as the only fermentable substrate. Previously, yeasts were subjected to starvation for $2 \mathrm{~h}$ in sterile saline solution $(0.9 \% \mathrm{NaCl})$ to remove any accumulated nutrients. Growth was monitored at $28{ }^{\circ} \mathrm{C}$ for 7 days in an automated spectrophotometer model Bioscreen C (Labsystem, Finland) by OD measurements. Maximum specific growth rate $\left(\mu_{\max }\right)$ in the presence of oleuropein was obtained from yeast growth curves by primarily modelling using the reparametized Gompertz equation proposed by Zwietering et al. (1990). For all tests described above, Saccharomyces boulardii was used as internal control.

\subsection{Evaluation of probiotic potential}

The following probiotic in vitro assays were also carried out (in triplicate) to the representative isolates obtained from each different cluster of the dendrogram, using the well-known probiotic yeast $S$. boulardii (Ultra Levura, Zambon S.A.U, Barcelona, Spain) as control: i) sequential simulated gastric and pancreatic digestion was performed according to the procedure described by Bonatsou et al. (2015), ii) the ability to remove cholesterol was determined in YNB as basal medium supplemented with cholesterol (182 mg/L) + glucose (10 g/L), cholesterol+glucose+oxgall (3 g/L) (SigmaAldrich), and cholesterol+oxgall, previously subjecting yeasts to starvation in $0.9 \%$ sterile saline solution for $2 \mathrm{~h}$. The purpose of employing three different media was to determine if the presence of different compounds could stimulate or inhibit cholesterol removal by yeasts. Cholesterol was quantified by absorbance at $500 \mathrm{~nm}$ and further 
comparison with a previous calibration curve (data not shown). All reagents were included in the Biosystem Kit (Barcelona, Spain); iii) biofilms assays on abiotic surfaces was carried out with the protocol described by Toledo-Arana et al. (2001). This ability was evaluated by quantification of the biomass production on the bottom of 96 microtiter plate wells by staining with crystal violet and measurements of absorbance at $595 \mathrm{~nm}$ in a Spectrostar Nano (BMG Labtech, Germany) spectrophotometer. The ability of the different yeast biotypes to form biofilms was measured alone and in co-culture with L. pentosus TOMC-LAB2 obtained from the Table Olive Microorganisms Collection (Instituto de la Grasa, CSIC, Spain). To assay biofilm formation in monocultures, L. pentosus and the different yeast strains were grown overnight at $30^{\circ} \mathrm{C}$ in MRS broth (Oxoid Unipath Ltd., Basingstoke, Hampshire, England) and YM broth, respectively. Next, the cells were diluted 1:40 in fresh medium, and $200 \mu \mathrm{L}$ of the bacterial or yeast suspension was grown in sterile 96-well Nunclon Delta surface microtiter plates (Nunc A/S, Denmark). For biofilm formation in coculture, L. pentosus or yeast cultures were grown as described above, and after dilution in fresh medium, $100 \mu \mathrm{L}$ of $L$. pentosus and $100 \mu \mathrm{L}$ of each yeast biotype suspension were inoculated together in sterile 96-well microtiter plates. After inoculation, both mono- and cocultures were incubated for 7 days at $30^{\circ} \mathrm{C}$, and then the biomass in biofilm was quantified. Measurements were performed by six times.

To investigate whether the formation of yeast-bacteria biofilms was mediated by a direct contact between microorganisms, a co-culture experiment on a 24-well polystyrene plate (CELLSTAR, Greiner Bio-One, Monroe, North Carolina, USA) was performed using L. pentosus TOMC-LAB2 and Candida boidinii Cb60 or Cb18, according to procedures described by Leon-Romero et al. (2016). Briefly, a $0.4 \mu \mathrm{M}$ pore size membrane (ThinCerts ${ }^{\mathrm{TM}}$ - TC Insert membrane, Greiner Bio-One) was placed 
within each one of the 24 wells of the plate to obtain a two-compartment device. The lower compartment was inoculated with $200 \mu \mathrm{L}$ of an overnight culture of $C$. boidinii (strain $\mathrm{Cb} 18$ or Cb60) and taken to a final volume of $2 \mathrm{~mL}$ with YM broth. The upper compartment was inoculated with $200 \mu \mathrm{L}$ overnight culture of L. pentosus TOMCLAB2 and filled up to $2 \mathrm{~mL}$ with MRS broth (Oxoid, Basingstoke, Hampshire, England). The plates were incubated at $28{ }^{\circ} \mathrm{C}$ for $48 \mathrm{~h}$. Subsequently, biofilms were observed under scanning electron microscope by the method described from Leon Romero et al. (2016). For this purpose, the samples were coated with gold and observed using a Jeol JSM-6460LV scanning electron microscope (Jeol USA, Inc., Peabody, MA) at the Centro de Investigacion, Tecnologia, e Innovacion de la Universidad de Sevilla (CITIUS, Seville, Spain).

\subsection{Statistical analysis}

To determine statistical differences among yeast strains subjected to the diverse technological and probiotic trials, analysis of variance (ANOVA) followed by posthoc comparison Scheffé test was applied. In a second step, to select the yeast biotypes with the best desirable technological and probiotic properties, multivariate analysis based on Principal Component Analysis (PCA) was applied. The Kaiser criterion was used to select the number of Principal Components (PCs), and only PC with eigenvalues higher than 1.00 were kept. Variables considered for inclusion in analysis were: percentage of isolation (Isolation), growth in presence of oleuropein (Oleu), cellular lipase (L-Ce), extracellular lipase (L-Ex), cellular esterase (E-Ce), extracellular esterase (E-Ex), cellular $\beta$-glucosidase (B-Ce), extracellular $\beta$-glucosidase (B-Ex), resistance (MIC) and susceptibility (NIC) to $\mathrm{NaCl}$, survival to gastric (GD) and pancreatic (PD) digestions, removal of cholesterol (RC) from YNB medium supplemented with cholesterol+glucose (RC-CG), cholesterol+glucose+oxgall (RC-CGO), and cholesterol+oxgall (RC-CO), 
ability to produce biofilm alone (auto-aggregation) or in presence of L. pentosus TOMC-LAB2 (co-aggregation). The cases introduced were the eight representative yeast isolates selected plus the reference probiotic control S. boulardii. Statistica software version 7.1 (StatSoft Inc, OK, Tulsa, USA) was used for data processing, analysis and generation of graphics.

\section{Results and discussion}

\subsection{Molecular study of the yeast population in Bosana olive brines}

In this work, a total of 72 yeast isolates obtained from Bosana naturally fermented black olives were first genotyped by RAPD-PCR analysis with M13 primer and further identified by sequencing of D1/D2 domains of 26S rDNA gene. First, reproducibility of RAPD technique was tested using a strain of W. anomalus TOMCY45 as an internal control, obtaining a reproducibility of $85.6 \%$, which was assumed as the cut-off value for selection of the different clusters and biotypes as described below. This value is between 80.5 and $90.0 \%$ obtained by Benítez-Cabello et al. (2015) and Tofalo et al. (2013), respectively, for other table olive related yeast species using the same technique. Many yeast studies use first a rapid screening procedure consisting of clustering the yeast isolates by fingerprinting methods, followed then by identification and further tests only with selected representatives of each cluster. Clustering by RAPD-PCR with primer M13 has been previously used with yeast related with oleic ecosystems (Tofalo et al. 2013; Benitez-Cabello et al., 2015; Mari et al., 2016). The risk of this methodology is to assume that all isolates grouped in the same cluster using only one primer belong to the same species or strain. For this reason, different primers for fingerprinting are usually applied for the same yeast population, especially for wine 
yeasts (Ramirez-Castrillón et al., 2014) or cured meat products (Andrade et al., 2006), although the degree of discrimination depends highly on the primer used.

The different profiles generated by the RAPD-PCR analysis ranged from a minimum of 2 to a maximum of 6 bands, and sizes of amplified fragments between 400 and 1,650 bp. The clustering analysis showed a total of seven groups above the selected cut-out value of $85.6 \%$ (Figure 1). Cluster I included a total of 28 yeast isolates (sharing $90.3 \%$ of similarity among them) all belonging to $W$. anomalus species as it was further confirmed by the sequencing of D1/D2 domains of $26 \mathrm{~S}$ rDNA gene. Cluster III $(84.7 \%$ of similarity), comprised 27 yeasts ascribed to Nakazawaea molendini-olei species as confirmed by sequencing and Blast analysis. The third group was Cluster V $(94.8 \%$ of similarity), which was composed by six isolates belonging to Candida boidinii species. Cluster IV was formed by five isolates assigned by the 26S rDNA sequencing to $C$. diddensiae ( $95.7 \%$ of similarity), while cluster VI was formed by four isolates of Zygotorulaspora mrakii sharing $97.7 \%$ of similarity among them. Finally, there were three clusters (II, VII, and VIII) formed by specific singleton biotypes belonging to Candida diddensiae, $C$. boidinii and $S$. cerevisiae species, as it was confirmed by D1/D2 domains 26S rDNA sequencing. Table 1 shows the frequency of isolates by biotype, with $W$. anomalus (38.8\%) and $N$. molendini-olei $(36.2 \%)$ as the most representative species of the yeast community from Bosana table olive fermentations. As clearly deduced from Fig. 1 and Table 1, it was found two different biotypes below the cut-off value of $85.6 \%$ for species $C$. boidinii and $C$. diddensiae. Tofalo et al. (2013) and Benitez-Cabello et al. (2015) used RAPD analysis with M13 primer for the study of the yeast populations obtained from different olive cultivars of Italian and Spain, respectively. In agreement with these authors, this work proved the efficacy of this technique as a useful tool to discriminate yeasts at the strain level. 
The presence of yeasts during processing of table olives around the world is very common, particularly species of the genera Candida, Pichia, Rhodotorula, Saccharomyces and Debaryomyces (Garrido-Fernández et al., 1997; Arroyo-López et al., 2012). This work describes for the first time the yeast community associated to fermentation of Bosana fruits. W. anomalus and N. molendini-olei species represented the $75 \%$ of isolates, with only a biotype for each species. The presence of W. anomalus in directly brined olives, and in table olives in general, is very common (Hernández et al., 2007; Bautista-Gallego et al., 2011; Arroyo-López et al., 2012; Mucilli et al., 2011; Doulgeraki et al., 2013; Mateus et al., 2016). N. molendini-olei was detected for the first time by Čadež et al. (2012) and Mari et al. (2016) in virgin olive oil and its products. Only recently, this species has been observed at low frequency in cracked green table olives by Mateus et al. (2016). Other important yeast species, but in this case detected at lower frequencies, was $C$. boidinii, which showed two different biotypes. The presence of $C$. boidinii in all table olives preparations is also very common. Arroyo-Lopez et al. (2008) referred presence of $C$. boidinii both in green and black directly brined olives. Farris et al. (1986) isolated some strains of $C$. boidinii in directly brined black olives, while Pereira et al. (2015) reported recently the presence of this species in the spontaneous fermentation of green olives.

\subsection{Technological characterization of the yeast biotypes}

The application of yeasts as starter cultures to lead the fermentation process of different table olive elaborations has been evaluated in the last years at pilot and industrial scale with promising results. Tufariello et al. (2015) used autochthonous strains of S. cerevisiae, W. anomalus (also known as Pichia anomala) and Debaryomyces hansenii in combination with LAB (Lactobacillus plantarum and 
Leuconostoc mesenteroides) to conduct the fermentation process. A significant decrease of the fermentation period and a significant improvement in the organoleptic characteristics of the final product were obtained for the production of fermented black table olive of different Italian and Greek cultivars. Recently, De Angelis et al. (2015) used an "omics" approach for evaluating the ability of selected autochthonous lactobacilli (L. plantarum) and yeasts (W. anomalus) to improve the fermentation process of Bella di Cerignola table olives processed as natural green olives. As shown through microbiological, biochemical, and sensory analyses, an accelerated fermentation was achieved using the selected lactobacilli and yeast strains. Coinoculation of $L$. pentosus and $C$. diddensiae was also favourable for the fermentation process of Arberquina natural green table olives, finding that the use of yeast reduced the Enterobacteriaceae survival period in comparison with the spontaneous process (Hurtado et al., 2010). All these data support the application of yeasts as starters for inoculation of table olive fermentations and justify the need for more detailed and specific studies to determine their technological features for selecting the most appropriated strains.

One representative strain from the different clusters obtained was randomly chosen and then subjected to diverse quantitative tests to determine their biotechnological potential. Table 2 summarizes the $\beta$-glucosidase, esterase, and lipase enzymatic activities obtained for the selected yeast strains, in both cellular and extracellular fractions. Most of the selected biotypes exhibited $\beta$-glucosidase activity in both fractions, with the exception of $C$. diddensiae $\mathrm{Cd} 7$ and $C$. boidinii $\mathrm{Cb} 18$ (cellular fraction) biotypes. The highest $\beta$-glucosidase value, in both cellular and extracellular fraction, was obtained for S. cerevisiae Sc24 strain, with higher values ( $p>0.05)$ than for the rest of yeasts. All yeasts strains showed esterase activity in both cellular and 
extracellular fractions. Values in the cellular fraction ranged from 39.50 (N. molendiniolei $\mathrm{Nm} 6)$ to $1,790.82 \mathrm{nmol} \mathrm{mL}^{-1} \mathrm{~h}^{-1}$ (W. anomalus Wa1), while values of the extracellular fraction ranged from 15.04 (C. boidinii Cb60) to $910.62 \mathrm{nmol} \mathrm{mL} \mathrm{H}^{-1} \mathrm{~h}^{-1}(W$. anomalus Wa1), with significant differences $(\mathrm{p}>0.05)$ between extreme values. With regard to lipase activity, W. anomalus Wa1, C. diddensiae $\mathrm{Cd} 7$ and C. boidinii $\mathrm{Cb} 18$ were the only biotypes which exhibited this activity in the cellular fraction, while in the extracellular fraction only $W$. anomalus Wa1, N. molendini-olei Nm6, and $C$. diddensiae Cd15 showed activity, being the highest significant value for W. anomalus Wa1 $(95.65 \mathrm{nmol} \mathrm{ml/h})$.

Several studies have been previously conducted to determine the enzymatic activities of table olive-related yeasts (Hernandez et al., 2007; Rodriguez-Gomez et al., 2010; Bautista-Gallego et al., 2011; Bevilacqua et al., 2013; Bonatsou et al., 2015). Production of esterase and lipase enzymes is an important feature for the breakdown of free fatty acids producing volatile components that could affect both flavour and aroma of table olives. In this study, W. anomalus Wa1 showed the highest esterase and lipase activities. Bonatsou et al. (2015) reported similar results for isolates of this species obtained from Greek black table olives. In general, production of lipase by the assayed yeast strains was very low. $C$. diddensiae $\mathrm{Cd} 7$ and $C$. boidinii $\mathrm{Cb} 18$ also showed a weak lipase activity. The presence of lipase seems to be a widespread feature of $C$. boidinii species (Rodríguez-Gómez et al. 2010), but in this study, the strain C. boidinii Cb60 did not exhibit this activity. The result is in agreement with Ciafardini et al. (2006) that described a negative lipase strain of $C$. boidinii isolated from extra virgin olive oil. On the contrary, esterase activity is a widespread property of yeast species isolated from table olives. Bautista-Gallego et al. (2011) found this activity in strains of $C$. diddensiae and W. anomalus. Esterase production by S. cerevisiae isolated from table olives was 
also reported by Hernandez et al. (2007). On the contrary, this feature was not found in S. cerevisiae strains associated to table olives by Silva et al. (2011) and Tofalo et al. (2013). Esterase and $\beta$-glucosidase activities could be involved in the hydrolysis of oleuropein and debittering of fruits. Several authors described high $\beta$-glucosidase activity by diverse strains of $W$. anomalus isolated from table olives (Bautista-Gallego et al. 2011; Bevilacqua et al., 2013; Tofalo et al. 2013; Bonatsou et al., 2015).

To determine the susceptibility (NIC) and resistance (MIC) of the selected yeast strains to $\mathrm{NaCl}$, a total of 351 growth curves were obtained and modelled by the use of a dose-response model. This methodology was also used by Bonatsou et al. (2015) to determine the resistance and susceptibility of Greek table olive-related yeasts to $\mathrm{NaCl}$. The ANOVA analysis, carried out with the MIC and NIC values obtained from triplicate experiments, showed that NIC values ranged from $24.5 \mathrm{~g} / \mathrm{L}$ (S. boulardii used as control) to 114.8 (W. anomalus Wa1), while MIC values ranged from $86.3 \mathrm{~g} / \mathrm{L}$ ( $S$. cerevisiae Sc24) to $171.5 \mathrm{~g} / \mathrm{L}$ (W. anomalus Wa1) (Table 2). Clearly, the biotype with the highest resistance to salt $(\mathrm{p}>0.05)$ was $W$. anomalus Wa1, while strains of $S$. boulardii and S. cerevisiae Sc24 were more sensitive to the use of salt. Data obtained for W. anomalus Wa1 are in agreement with previous studies which determined the resistance to $\mathrm{NaCl}$ of strains of this species, which reported MIC values ranging from 163 to 167 g/L (Romero-Gil et al., 2013; Bonatsou et al., 2015). However, data obtained for $S$. cerevisiae Sc24 were lower than those found in the bibliography, with MIC values ranging from 163 to 274 g/L (Romero-Gil et al., 2013; Bonatsou et al., 2015). For many of the yeast species assayed in this work (C. diddensiae, N. molendini-olei, and $Z$. mrakii) these type of data have been obtained for the first time. 
Oleuropein is the polyphenol responsible for the bitter taste of olive fruits, and its biological hydrolysis is a very important feature for the debittering process in no "lye"-treated olives. In this study, the growth of selected biotypes in the presence of a basal medium supplemented with $1 \%$ oleuropein was modelled through a total of 54 growth curves and the use of a sigmoid primary model. Most of the strains showed low values of $\mu_{\max }$ in the presence of oleuropein. However, strain W. anomalus Wa1 exhibited the highest $\mu_{\max }$ value $\left(0.32 \mathrm{~h}^{-1}\right)$, statistically different $(\mathrm{p}>0.05)$ from the rest of strains (Table 2). On the contrary, strains Z. mrakii Zm14 and C. diddensiae Cd7 showed the lowest $\mu_{\max }$ values $\left(0.04 \mathrm{~h}^{-1}\right)$, thus indicating difficulty to growth in the presence of this polyphenol as the only substrate for growth. In the last few years, researchers have put their attention on the study of oleuropein degradation by yeasts for biological debittering of the fruits. In particular, Silva et al. (2011) found five strains of Candida oleophila with oleuropeinolytic activity while the ability to hydrolyze oleuropein by strains of $D$. hansenii and Torolospura delbrueckii was reported by Psani and Kotzekidou (2006).

\subsection{Probiotic potential of the yeast biotypes}

Few studies have evaluated specifically the probiotic potential of yeasts isolated from table olives (Silva et al., 2011; Bonatsou et al., 2015). Their use as starters in olive fermentations could also turn this fermented vegetable as a convenient carrier of beneficial yeasts to the human body. Usually, human gut has been the main source of probiotic strains but recent studies showed the probiotic features of microorganisms isolated from fermented foods. Moslehi-Jenabian et al. (2010) discussed the potential that food-borne yeasts could improve human health. Moreover, the attention of many researchers is currently focused on turning table olives as a carrier of probiotic 
microorganisms to the human body (Lavermicocca et al., 2005). Thus, the study of the probiotic potential of table olive related yeasts is of great interest.

Resistance to gastric and pancreatic juices secreted during passage through the digestive system is a very important feature of probiotic strains. For this purpose, the capability of yeast strains to survive in simulated gastric and pancreatic conditions was investigated. The results from both in vitro digestions are expressed in percentages of survival and reported in Table 3. S. boulardii, used as a probiotic yeast control, showed the highest survival to gastric digestion $(95.9 \%)$. However, diverse yeast biotypes $(S$. cerevisiae $\mathrm{Sc} 24$, Candida diddensiae $\mathrm{Cd} 7$ and $\mathrm{Cd} 15$, C. boidinii $\mathrm{Cb} 60$ and W. anomalus Wa1) did not show significant differences with respect to S. boulardii. In general, survival to simulated in vitro pancreatic digestions by the yeasts were lower as compared with gastric digestions (see Table 3). In this case, certain yeast biotypes ( $S$. cerevisiae Sc24, C. diddensiae $\mathrm{Cd} 7$ and $\mathrm{Cd} 15$, C. boidinii Cb60) exhibited even a higher survival compared to the S. boulardii (10.9\%) control. Similar results were achieved by Bonatsou et al. (2015) who reported a considerable resistance of table olive related yeasts to simulated gastric and pancreatic digestions. These results are also in accordance to the findings obtained by Pennacchia et al. (2008), who observed a high ability of strains of $S$. cerevisiae isolated from different foods to survive to environmental conditions simulating those observed into the gastrointestinal tract. On the contrary, Silva et al., (2011) reported low survival to acidic conditions of $S$. cerevisiae isolated from Portuguese table olives. Our data showed the substantial resistance to simulated gastric and pancreatic digestions of Bosana olive related yeasts.

The ability of $S$. boulardii and the other assayed yeasts to remove in vitro cholesterol in three different media was also tested. Results obtained from 
spectrophotometric assays revealed that the percentage of cholesterol reduction depends on the type of culture medium used. The medium where all yeasts showed the highest percentage of cholesterol reduction was YNB supplemented with cholesterol and glucose, with values ranging from 20.40 (C. boidinii Cb-60) to $26.30 \%$ (Z. mrakii Zm14) with significant differences between extreme values. On the contrary, the medium with the lowest percentage in cholesterol reduction was YNB supplemented with cholesterol/glucose/oxgall. Here the percentage of reduction ranged between 10.83 (S. boulardii) and $15.67 \%$ (C. boidinii $\mathrm{Cb18)}$ with no significant differences between extreme values (Table 3). In general, strains of C. boidinii Cb18 and S. cerevisiae Sc24 exhibited a great ability to degrade cholesterol in all culture media. These strains showed a higher percentage of cholesterol degradation even than the probiotic control $S$. boulardii. This is the first study that evaluates the ability of yeast strains isolated from table olives to remove cholesterol in vitro. Kourelis et al. (2010) described the ability of S. cerevisiae, S. boulardii and diverse Candida strains isolated from feta cheese or gastrointestinal tract of infants to reduce cholesterol levels in vitro, with values ranging from 4 to $60 \%$ of reduction for strains of the same species. Also, Şanlidere et al. (2015) observed that a low amount of lyophilized yeasts administered with animal feed reduced the level of cholesterol of rats at the rate of $25 \%$, similar to values obtained in this work.

The ability of the selected yeasts to form biofilms on abiotic surfaces (plastic) was evaluated in vitro alone and in co-culture with a L. pentosus strains previously isolated from a table olive processing (TOMC-LAB2). We have to take in consideration that with the methodology used we have no way to determine the specific contribution of the yeast and bacteria in the mixed biofilm. This way, we do not have information if most of the biofilms corresponds to the yeasts, the LAB strain or equally for both species. Results on biofilm quantification revealed that for many of the yeast strains 
used in this work biofilm production was higher in the presence of the LAB strain than in monoculture (Fig 2). In fact, yeast strains Z. mrakii Zm14, S. boulardii, N. molendiniolei Nm6, C. diddensiae Cd7, C. boidinii Cb18, and S. cerevisiae Sc24, showed a low ability to form biofilm in monoculture $(\mathrm{OD}<0.6)$, but considerably increased in presence of the LAB strain (especially evident in the cases of $S$. boulardii and $C$. boidinii $\mathrm{Cb} 18$ ). Only biotypes $W$. anomalus Wa1, C. diddensiae Cd15, and C. boidinii Cb60 showed a higher ability of biofilm production in monocultures, especially relevant for $C$. boidinii Cb60. The highest ability to form biofilm in mixed culture with L. pentosus TOMCLAB2 was noticed for $S$. boulardii, W. anomalus Wa1, and the two biotypes of $C$. boidinii. Leon-Romero et al. (2016) achieved similar results and proved that the best biofilm formation was noticed for $C$. boidinii and $W$. anomalus in mixed culture with $L$. pentosus, but not for other yeast species, and that this association can be inhibited by $\mathrm{D}$ $-(+)$ mannose. Data obtained in this work suggests that the yeast-lactobacilli biofilms may be favoured by the presence of a specific mate of yeast-L. pentosus. However, it should be confirmed that this ability to form biofilms by the selected strains on abiotic surfaces (microplates) is also obtained on biotic surfaces (fruit epidermis). This way, table olives could be turned on a carrier of beneficial microorganisms to the human body.

To determine if biofilm formation was mediated by direct contact between the C. boidinii and L. pentosus cells, both microorganisms were inoculated in different compartments of the same well separated by a membrane of $0.4 \mu \mathrm{M}$ pore size. After incubation, SEM technique was used to determine the morphology of the $C$. boidinii cells (Fig 3). Results showed that presence of filter does not affect biofilm development in $C$. boidinii $\mathrm{Cb18}$, obtaining a strong auto-aggregation of yeast cells even in the absence of direct contact with L. pentosus (Fig 3, lower panel). Therefore, the 
membrane allowed the diffusion of LAB metabolites that stimulated the formation of biofilms by $C$. boidinii. Accordingly, the formation of biofilm by $C$. boidinii $\mathrm{Cb} 18$ was lower in mono-cultures and in absence of LAB metabolites (Fig 3, upper panel). LeonRomero et al. (2016) also reported that biofilm formation by C. boidinii in monocultures was stimulated even in the absence of cell-cell contact with L. pentosus. It has been reported that the addition of cell-free supernatants from Lactobacillus casei subsp. rhamnosus IFO3831 also induced biofilm formation by S. cerevisiae Kyokai-10, a yeast-isolate from rice wine, in mono-culture (Kawarai et al. 2007).

\subsection{Selection of the most promising yeast biotypes}

The application of an appropriate methodology for the management of a large amount of data is necessary when researchers analyze several physiological traits (variables) from a considerable number of strains (cases). Multivariate analysis techniques offer an interesting approach to solve this drawback. In the last years, many researchers have used this methodology for the selection of the most promising yeast starters related with table olive processing (Bevilacqua et al., 2013; Rodriguez-Gómez et al., 2012; Bonatsou et al., 2015).

To reduce the number of original variables (17) into a smaller number of PCs, quantitative data obtained from technological and probiotic tests were subjected to multivariate analysis (PCA). Five eigenvalues higher of 1 were obtained, showing that the original variables can be grouped into five PCs that counted for the $92.5 \%$ of total variance. Fig. 4 (upper panel) shows the relation between the two major PCs and the original variables. As can be easily deduced from the graph, PC 1 has a negative correlation practically with all technological features (enzymatic activities, resistance and susceptibility to $\mathrm{NaCl}$, the frequency of isolation, and growth in the presence of 
oleuropein) and could then be referred as technical properties. On the contrary, PC 2 has a positive correlation practically with all probiotic features (resistance to simulated digestions, ability to form biofilm alone and in co-culture) except with the removal of cholesterol in certain media, and could be associated with health properties. The projection of cases (selected yeast biotypes plus probiotic control) onto the planes formed by the two major PCs (Fig 4, lower panel) explained the majority of variance $(55.5 \%)$ and allowed discrimination of yeast biotypes into three clear groups. Two groups were placed in the positive axis of PC 1, but in the positive (S. cerevisiae $\mathrm{Sc} 24$, C. boidinii Cb60, C. diddensiae $\mathrm{Cd} 15$ and $S$. boulardii) and negative (C. diddensiae Cd7, C. boidinii Cb18, Z. mrakii Zm14 and N. molendini-olei Nm6) axis of PC 2. The first group, where it was also included the probiotic yeast $S$. boulardii, can be assigned to yeasts with a high probiotic potential, especially strains $S$. cerevisiae $\operatorname{Sc} 24$ and $C$. boidinii $\mathrm{Cb60}$, while the second group was weak in both technological and probiotic features. Finally, there was a single isolate (W. anomalus Wa1) placed in the negative axis of PC 1, being clearly related with the highest technological potential.

$W$. anomalus is a yeast species well adapted to the environmental conditions that drive table olive fermentations such as low $\mathrm{pH}$ and high $\mathrm{NaCl}$ concentration (Arroyo et al., 2005). In a previous study, Hernández et al. (2007) found diverse strains of this species isolated from table olive fermentations with interesting technological applications. Recently, Bautista-Gallego et al. (2011) and Tofalo et al. (2013) reported the high $\beta$-glucosidase activity exhibited for this microorganism. All these features make this species one of the best candidates to obtain isolates with technological applications in table olives, as it was recently reported by Rodriguez-Gómez et al. (2012), Bevilacqua et al. (2013), and Bonatsou et al. (2015). Data obtained in this work prove again the high potential for technological applications exhibited for this species. 
Regarding the probiotic potential of isolates S. cerevisiae Sc24 and C. boidinii Cb60, this is the first time that isolates of these species present this ability, and further studies are necessary to corroborate these preliminary data.

\section{Conclusions}

The present study is the first report regarding yeast population associated to Bosana table olive fermentations. Results obtained by molecular methods show that two species (W. anomalus and N. molendini-olei) accounted for $75 \%$ of total isolates. The analysis in vitro of their multifunctional features, and the application of multivariate analysis techniques showed that certain isolates had promising perspective of use for technological (W. anomalus Wa1) or probiotic $(S$. cerevisiae $\mathrm{Sc} 24$ and $C$. boidinii Cb60) applications. However, these preliminary data should be validated in further studies at pilot or industrial scale.

\section{Acknowledgements}

The research leading to these results has received funding from the Spanish Government (Project OliFilm AGL-2013-48300-R: www.olifilm.science.com.es), and Junta de Andalucía (through financial support to projects P11-AGR-7051, P11-AGR7755, and groups AGR-125 and BIO-160). CP gratefully acknowledges Sardinia regional Government for the financial support of her PhD scholarship (POR Sardegna FSE OP European Social Fund 2007/2013 - Axis IV Human Resources, Objective 1.3, Line of activity 1.3.1) and the Foundation of Sardinia (Prat. 2013.1364) for financial support. FNAL wish to express thanks to the Spanish Government for his postdoctoral research contract (Ramón y Cajal). Authors express their gratitude to Dr. Antonio Garrido Fernández for his constructive comments and suggestions with drafting of 
manuscript. CP gratetfully acknowledges Dr Ivo Pinna for kindly provided support of her thesis manuscript.

\section{References}

Andrade, M.J., Rodríguez, M., Sánchez, B., Aranda, E., Córdoba, J.J., 2006. DNA typing methods for differentiation of yeasts related to dry-cured meat products. Int. J. Food Microbiol. 107, 48-58.

Andrighetto, C., Psomas, E., Tzanetakis, N., Suzzi, G., Lombardi, A., 2000. Randomly amplified polymorphic DNA (RAPD) PCR for the identification of yeasts isolated from dairy products. Lett. Appl. Microbiol. 30, 5-9.

Arroyo, F.N., Durán Quintana, M.C., Garrido Fernández, A., 2005. Evaluation of primary models to describe the growth of Pichia anomala and study of temperature, $\mathrm{NaCl}$, and $\mathrm{pH}$ effects on its biological parameters by response surface methodology. J. Food. Prot. 68, 562-570.

Arroyo-López, F. N., Querol, A., Bautista-Gallego, J., Garrido-Fernández, A., 2008. Role of yeasts in table olive production. Int. J. Food Microbiol. 128 189-196

Arroyo-Lopez, F. N., Duran-Quintana, M. C., Ruiz-Barba, J. L., Querol, A., GarridoFernandez, A., 2006. Use of molecular methods for the identification of yeast associated with table olives. Food Microbiol. 23(8), 791-796.

Arroyo-Lopez, F.N., Romero-Gil, V., Bautista-Gallego, J., Rodriguez-Gomez, F., Jimenez-Diaz, R., Garcia-Garcia, P., Querol, A., Garrido-Fernandez, A., 2012. Yeasts in table olive processing: Desirable or spoilage microorganisms? Int. J. Food Microbiol. 160(1), 42-49.

Arroyo-López, F.N., García-Gacía P., Rodríguez-Gómez F., Garrido-Fernández, A., 2016. Olives: types and consumption. In: The Encyclopedia of Food and Health vol. 4, pp. 167-170. Oxford: Academic Press.

Bach-Faig, A., Berry, E. M., Lairon, D., Reguant, J., Trichopoulou, A., Dernini, S., Medina, F. X., Battino, M., Belahsen, R., Miranda, G., Serra-Majem, L., 2011. 
Mediterranean diet pyramid today. Science and cultural updates. Public Health Nutr. 14(12A), 2274-2284

Bautista-Gallego, J., Rodríguez-Gómez, F., Barrio, E., Querol, A., Garrido-Fernández, A., Arroyo-López, F.N., 2011. Exploring the yeast biodiversity of green table olive industrial fermentations for technological applications. Int. J. Food Microbiol. 147, 89-96.

Benitez-Cabello, A., Romero-Gil, V., Rodriguez-Gómez, F., Garrido-Fernández, A., Jiménez-Díaz, R., Arroyo-López, F.N., 2015. Evaluation and identification of polymicrobial biofilms on natural green Gordal table olives. Antonie van Leeuwenhoek $108,597-610$.

Bevilacqua, A., Corbo, M.R., Sinigaglia, M., 2012. Selection of yeasts as starters cultures for table olives: a step-by-step procedure. Front. Microbiol. 31. Art. 194.

Bevilacqua, A., Beneduce, L., Sinigaglia, M., Corbo, M.R., 2013. Selection of yeasts as starters cultures for table olives. J. Food Sci. 78, 742-751.

Bevilacqua, A., de Stefano, F., Augello, S., Pignatiello, S., Sinigaglia, M., Corbo, M.R. 2015. Biotechnological innovations for table olives. Int J Food Sci Nutr. 66(2),12731.

Bleve, G., Tufariello, M., Durante, M., Grieco, F., Ramires, F.A., Mita, G., TasioulaMargari, M., Logrieco, A.F., 2015. Physico-chemical characterization of natural fermentation process of Conservolea and Kalamàta table olives and development of a protocol for the pre-selection of fermentation starters. Food Microbiol. 46, 368-82.

Bonatsou, S., Benitez, A., Rodriguez-Gomez, F., Panagou, E. Z. and Arroyo-Lopez, F. N., 2015. Selection of yeasts with multifunctional features for application as starters in natural black table olive processing. Food Microbiol. 46, 66-73.

Čadež, N., Raspor, P., Turchetti, B., Cardinali, G., Ciafardini, G., Veneziani, G., Peter, G., 2012. Candida adriatica sp. nov. and Candida molendinolei sp. nov., two yeast species isolated from olive oil and its by-products. Int. J. Syst. Evol. Micr. 62(9), 2296-2302. 
Ciafardini, G., Zullo, B. A., Cioccia, G., Iride, A., 2006. Lipolytic activity of Williopsis californica and Saccharomyces cerevisiae in extra virgin olive oil. Food Microbiol. 107, 27-32.

Corsetti, A., Perpetuini, G., Schirone, M., Tofalo, R., Suzzi, G., 2012. Application of starter cultures to table olive fermentation: An overview on the experimental studies. Front. Microbiol. 3, 1-6.

De Angelis, M., Campanella, D., Cosmai, L., Summo, C., Rizzello, C.G., Caponio, F., 2015. Microbiota and metabolome of un-started and started Greek-type fermentation of Bella di Cerignola tableolives. Food Microbiol. 52,18-30.

Doulgeraki, A. I., Pramateftaki, P., Argyri, A. A., Nychas, G. J. E., Tassou, C. C., Panagou, E. Z., 2013. Molecular characterization of lactic acid bacteria isolated from industrially fermented Greek table olives. LWT - Food Sci. Technol. 50, 353356

Farris, G. A., Forteleoni, M., Pais, L., 1986. I lieviti delle salamoie delle olive da mensa. Annali della Facoltà di Agraria dell’Universita di Sassari.

Garrido-Fernandez, A., Fernandez-Diez, M. J., Adams, M. R., 1997. Table olives: production and processing, Chapman \& Hall., 496 p.

Hernández, A., Martin, A., Aranda, E., Pérez-Nevado, F., Córdoba, M.G., 2007. Identification and characterization of yeast isolated from the elaboration of seasoned green table olives. Food Microbiol. 24, 346-351

Hurtado, A., Reguant, C., Bordons, A., Rozès, N., 2010. Evaluation of a single and combined inoculation of a Lactobacillus pentosus starter for processing cv. Arbequina natural green olives. Food Microbiol. 27(6), 731-740.

IOC (International Olive Oil Council). 2017. World table olives figures. http://www.internationaloliveoil.org/estaticos/view/132-world-table-olive-figures. Last access: February 2017. 
Kawarai, T., Furukawa, S., Ogihara, H., Yamasaki, M., 2007. Mixed-species biofilm formation by lactic acid bacteria and rice wine yeasts. Appl Environ Microbiol 73, 4673-4676.

Kourelis, A., Kotzamanidis, C., Litopoulou-Tzanetaki, E., Scouras, Z. G., Tzanetakis, N., Yiangou, M., 2010. Preliminary probiotic selection of dairy and human yeast strains. J. Biol. Res. 13, 93-104.

Kurtzman, C. P. and Robnett, C. J., 1998. Identification and phylogeny of ascomycetous yeasts from analysis of nuclear large subunit (26S) ribosomal DNA partial sequences. Antonie van Leeuwenhoek. 73, 331-371.

Lavermicocca, P., Valerio, F., Lonigro, S. L., De Angelis, M., Morelli, L., Callegari, M. L., Rizzello, C. G., Visconti, A., 2005. Study of adhesion and survival of lactobacilli and bifidobacteria on table olives with the aim of formulating a new probiotic food. Appl. Environ. Microbiol. 71(8), 4233-4240.

Leon-Romero, A., Dominguez-Manzano, J., Garrido-Fernandez, A., Arroyo-Lopez, F. N., Jimenez-Diaz, R., 2016. Formation of In Vitro Mixed-Species Biofilms by Lactobacillus pentosus and Yeasts Isolated from Spanish-Style Green Table Olive Fermentations. Appl. Environ. Microbiol. 82(2), 689-695.

Mari, E., Guerrini, S., Granchi, L., 2016. Enumeration and rapid identification of yeasts during extraction processes of extra virgin olive oil in Tuscany. World J. Microb. Biot. 32(6), 1-10.

Martorana, A., Alfonzo, A., Gaglio, R., Settanni, L., Corona, O., La Croce, F., Vagnoli, P., Caruso, T., Moschetti, G., Francesca, N., 2017. Evaluation of different conditions to enhance the performances of Lactobacillus pentosus OM13 during industrial production of Spanish-style table olives. Food Microbiol. 61, 150-158.

Mateus, T., Santo, D., Sade, C., Pires-Cabral, P., Quintas, C., 2016. The effect of NaCl reduction in the microbiological quality of cracked green table olives of the Maanilha Algarvia cultivar. Int. J. Food Microbiol. 218, 57-65. 
Moslehi-Jenabian, S., Lindegaard Pedersen, L., Jespersen, L., 2010. Beneficial effects of probiotic and food borne yeasts on human health. Nutr. 2, 449-473.

Muccilli, S., Caggia, C., Randazzo, C. L., Restuccia, C., 2011. Yeast dynamics during the fermentation of brined green olives treated in the field with kaolin and Bordeaux mixture to control the olive fruit fly. Int. J. Food Microbiol. 148(1), 15-22.

Pennacchia, C., Blaiotta, G., Pepe, O., Villani, F., 2008. Isolation of Saccharomyces cerevisiae strains from different food matrices and their preliminary selection for a potential use as probiotics. J. Appl Microbiol. 105, 1919-1928.

Pereira, D. I., Gibson, G. R., 2002. Cholesterol Assimilation by Lactic Acid Bacteria and Bifidobacteria Isolated from the Human Gut Cholesterol Assimilation by Lactic Acid Bacteria and Bifidobacteria Isolated from the Human Gut. Appl. Environ. Microbiol. 68(9), 1-6.

Pereira, E.L., Ramalhosa, E., Borges, A., Pereira, J.A., Baptista, P., 2015. Yeast dynamics during the natural fermentation process of table olives (Negrinha de Freixo cv.). Food Microbiol. 46,582-586.

Pinna, I. 2005. Evoluzione chimico-fisica e microbiologica di olive nere da mensa fermentate in salamoia al naturale. $\mathrm{PhD}$ tesi, University of Sassari. Bibl. Nazionale Centrale Firenze TDR 2005 4385, CF980504385.

Psani, M. and Kotzekidou, P., 2006. Technological characteristics of yeast strains and their potential as starter adjuncts in Greek-style black olive fermentation. World J. Microb. Biot. 22(12), 1329-1336.

Ramírez-Castrillón, M., Camargo-Mendes, S.D., Inostroza-Ponta, M., Valente, P., 2014. $(\mathrm{GTG})_{5}$ MSP-PCR fingerprinting as a technique for discrimination of wine associated yeasts?. PlosOne 9:e105870.

Rodriguez-Gomez, F., Arroyo-Lopez, F. N., Lopez-Lopez, A., Bautista-Gallego, J., Garrido-Fernandez, A., 2010. Lipolytic activity of the yeast species associated with the fermentation/storage phase of ripe olive processing. Food Microbiol. 27(5), 604612. 
Rodríguez-Gómez, F., Romero-Gil, V., Bautista-Gallego, J., Garrido-Fernández, A., Arroyo-López, F.N., 2012. Multivariate analysis to discriminate yeast strains with technological applications in table olive processing. World J. Microb. Biot. 28, 1761-1770.

Rodríguez-Gómez, F., Romero-Gil, V., Arroyo-López, F.N., Roldán-Reyes, J., TorresGallardo, R., Bautista-Gallego, J., García-García, P., Garrido-Fernández, A., 2017. Assessing the challenges in the application of potential probiotic lactic acid bacteria in the large-scale fermentation of Spanish style table olives. Frontiers Microbiol. 8, 915.

Romero-Gil, V., Bautista-Gallego, J., Rodriguez-Gomez, F., Garcia-Garcia, P., Jimenez-Diaz, R., Garrido-Fernandez, A., Arroyo-Lopez, F. N., 2013. Evaluating the individual effects of temperature and salt on table olive related microorganisms. Food Microbiol. 33(2), 178-184.

Ruiz-Barba, J. L. and Jimenez-Diaz, R., 1995. Availability of essential B-group vitamins to Lactobacillus plantarum in green olive fermentation brines. Appl. Environ. Microbiol. 61(4), 1294-1297.

Ruiz-Barba, J.L., Brenes, M., Jiménez Díaz, R., García, P., Garrido, A., 1993. Inhibition of Lactobacillus plantarum by polyphenols extracted from two different kinds of olive brines. J. Appl. Bacteriol. 74, 15-19.

Şanlidere, H., Ezgi, A., Ozer, D., 2015. Investigation of a Probiotic Yeast as a Cholesterol Lowering Agent on Rats Fed on a High Cholesterol Enriched Diet [ 1 ] Probiyotik Mayanın Yuksek Kolesterol İceren Diyetle Beslenen Sicanlarda Kolesterolu Duşurucu Ajan Olarak Kullanılabilirliğinin Araştır', 21(5), 685-689.

Senses-Ergul, S., Karasu-Yalcin, S., Ozbas, Z.Y., 2012. Molecular identification of some yeast strains isolated from various sugary foods. Ann. Microbiol. 62, 15011506.

Silva, T., Reto, M., Sol, M., Peito, A., Peres, C.M., Peres, C., Xavier-Malcata, F., 2011. Characterization of yeasts from Portuguese brined olives, with a focus on their potentially probiotic behavior. LWT-Food Sci. Technol. 44, 1349-1354. 
Tassou, C.C., Panagou, E.Z., Katsaboxakis, K.Z., 2002. Microbiological and physicochemical changes of naturally black olives fermented at different temperatures and $\mathrm{NaCl}$ levels in the brines. Food Microbiol. 19(6), 605-615.

Tofalo, R., Perpetuini, G., Schirone, M., Suzzi, G., Corsetti, A., 2013. Yeast biota associated to naturally fermented table olives from different Italian cultivars. Inte. J. Food Microbiol. 161, 203-208.

Tofalo, R., Perpetuini, G., Schirone, M., Ciarrochi, A., Faroli, G., Suzzi, G., Corsetti, A., 2014. Lactobacillus pentosus dominates spontaneous fermentation of Italian table olives. LWT Food Sci. Technol. 57, 710-717.

Toledo-Arana, A., Valle, J., Solano, C., Arrizubieta, M.J., Cucarella, C., Lamata, M., Amorena, B., Leiva, J., Penadés, J.R., Lasa, I., 2001. The enterococcal surface protein, Esp, is involved in Enterococcus faecalis biofilm formation. Appl. Environ. Microbiol., 67, 4538-4545

Tufariello M, Durante M, Ramires FA, Grieco F, Tommasi L, Perbellini E, Falco V, Tasioula-Margari M, Logrieco AF, Mita G, Bleve G., 2015. New process for production of fermented black table olives using selected autochthonous microbial resources. Front Microbiol. 6,1007.

Zwietering, M.H., Jongenburger, I., Rombouts, F.M., Van't Riet, K., 1990. Modeling of the bacterial growth curve. Appl. Environ. Microbiol. 56, 1875-1881. 


\section{Figure legends}

Fig 1. Dendrogram generated after bioinformatic analysis with Bionumerics 6.6 software package of the RAPD-PCR profiles obtained with M13 primer for the 72 yeast isolates randomly obtained from Bosana olive brines. The red asterisks indicate representative isolates randomly selected from the different clusters (in Roman numerals) for the technological and probiotic in vitro tests. Strain W. anomalus TOMCY45 was used as internal control to assess the reproducibility of the technique. S.b: stands for the fingerprint of $S$. boulardii used as a probiotic control.

Fig 2. Biofilm formation (expressed as OD values at $595 \mathrm{~nm}$ ) of the different yeast genotypes obtained in the present study alone and in co-cultures with L. pentosus TOMC-LAB2. Vertical bars indicate standard error. $S b$ stands for $S$. boulardii used as a probiotic control. The OD value of $L$. pentosus TOMC-LAB2 in mono-culture was $1.27 \pm 0.14$.

Fig 3. Scanning electron microscopy of $C$. boidinii $\mathrm{Cb} 18$ cells in YM broth alone (upper panel) and in microtiter compartmentalized wells with $0.4 \mu$ size pore in the presence of L. pentosus TOMC-LAB2 (lower panel).

Fig 4. PCA analysis of the yeast genotypes obtained in the present study as a function of the different technological and probiotic features. A) Projection of the variables onto the plane formed by the first two major PCs, B) Projection of the yeast biotypes onto the planed formed by the two major PCs. For acronym of variables, please see material and method section. 
Figure 1
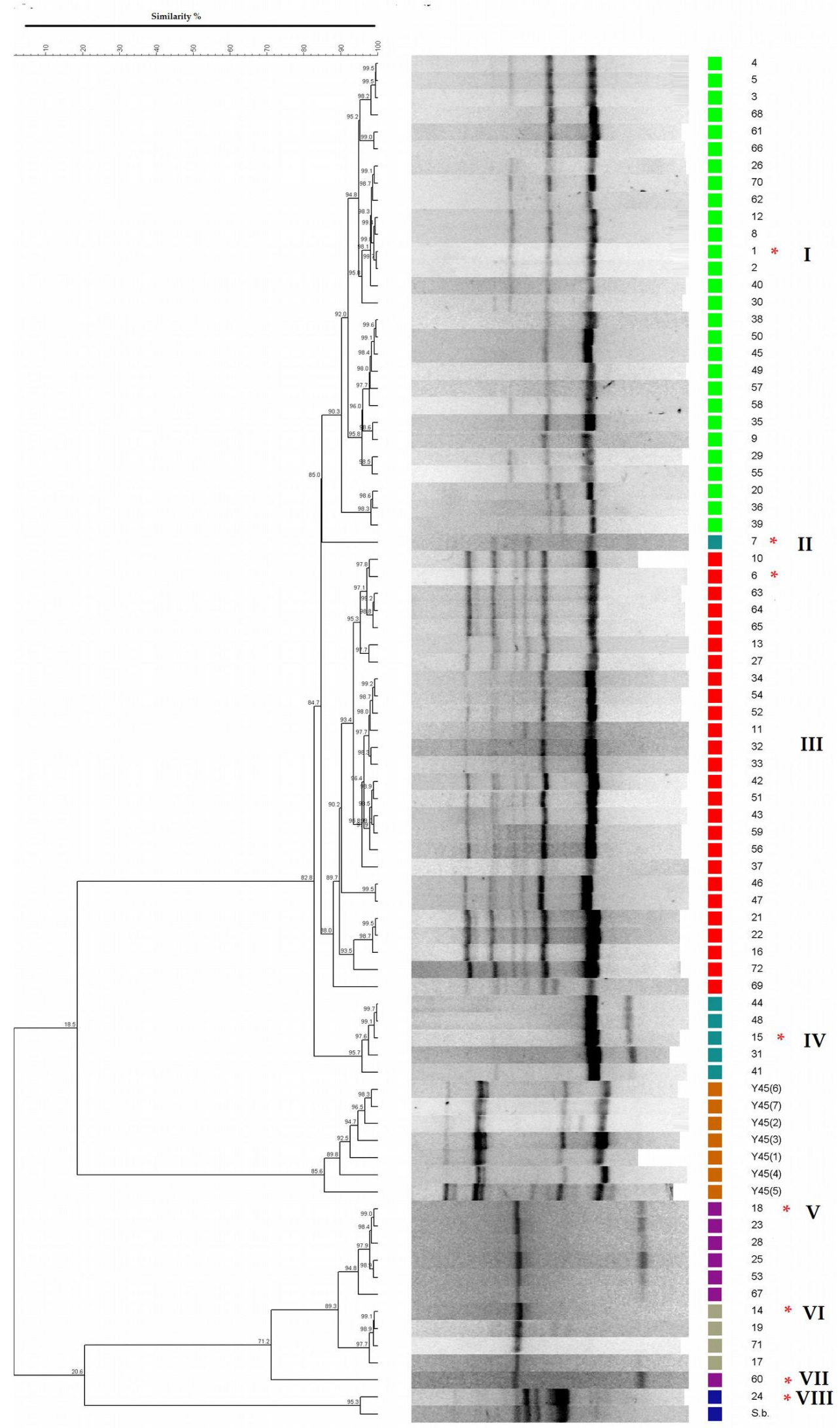
Figure 2

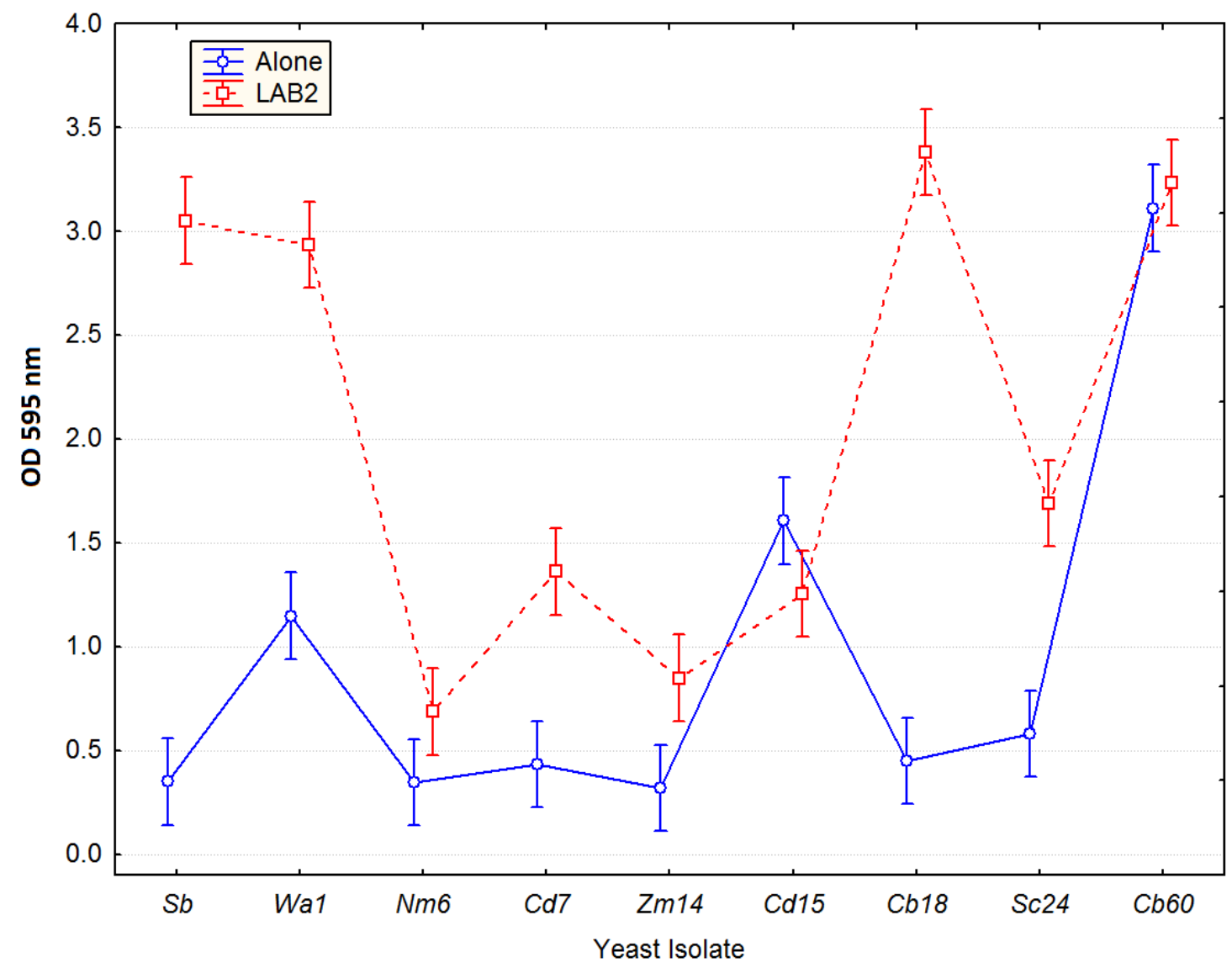


Figure 3
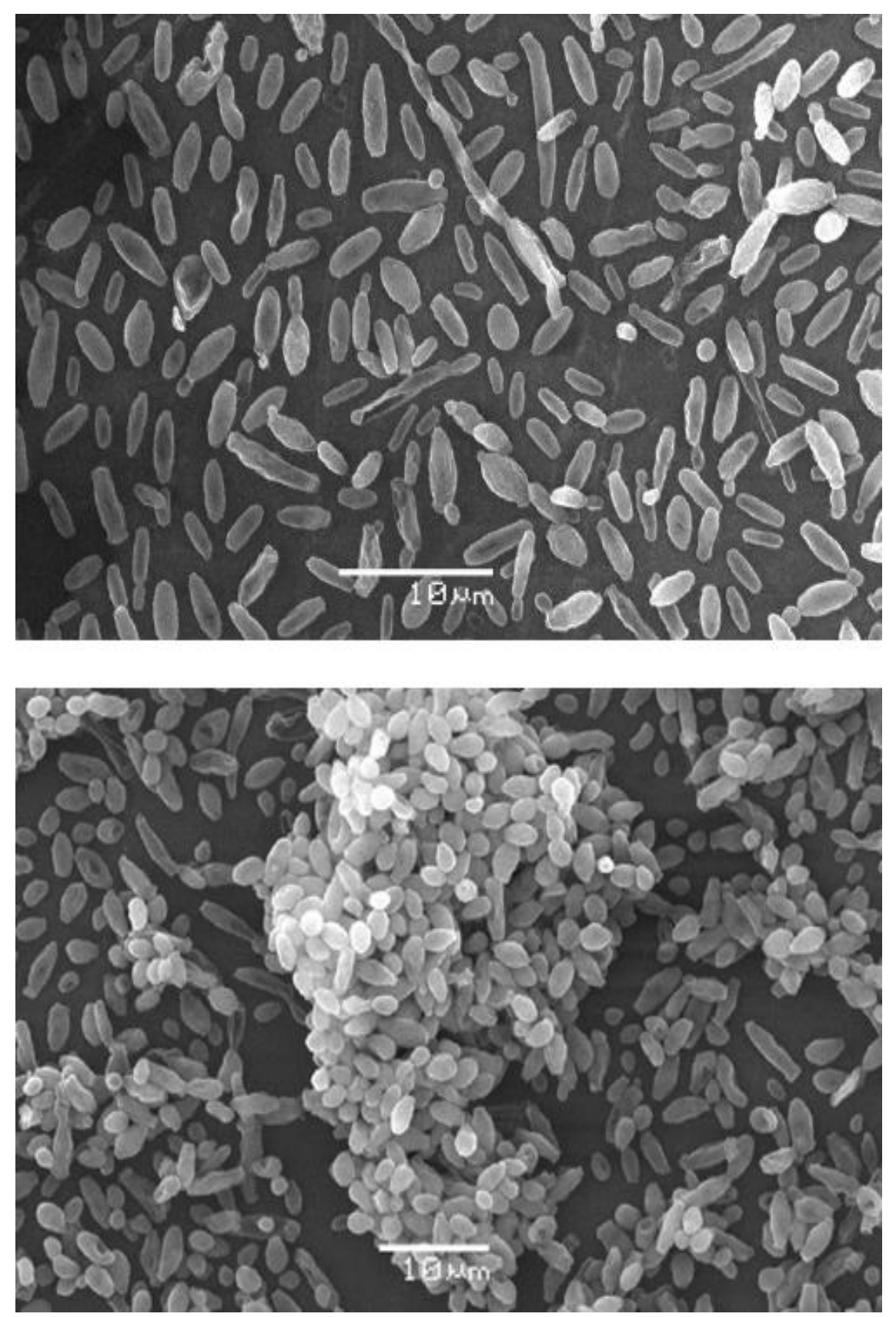
Figure 4
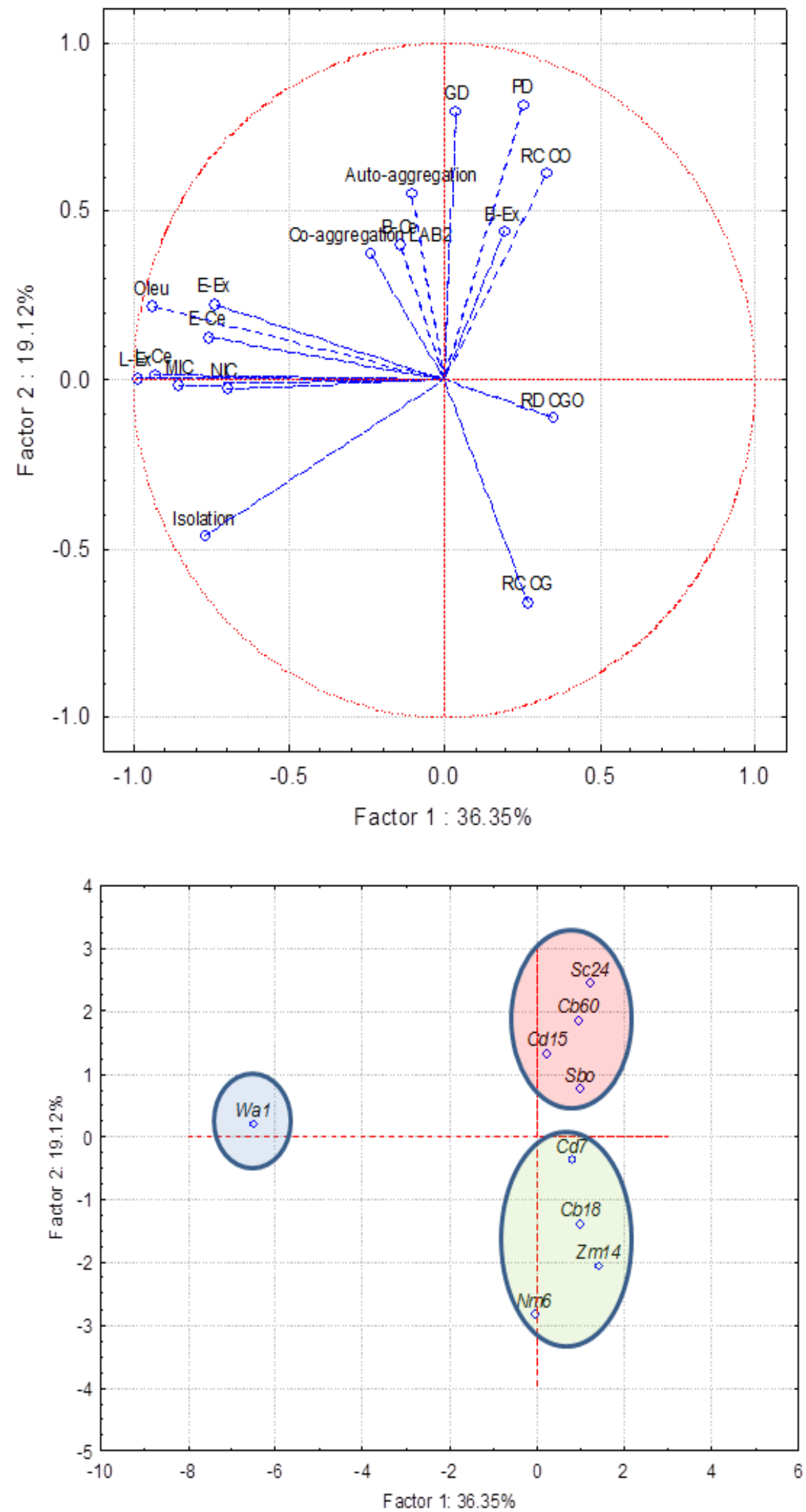